\title{
QT interval dispersion in chronic heart failure and left ventricular hypertrophy: relation to autonomic nervous system and Holter tape abnormalities
}

\author{
P P Davey, J Bateman, I P Mulligan, C Forfar, C Barlow, G Hart
}

\begin{abstract}
Objective-To study QT dispersion in left ventricular hypertrophy and chronic heart failure and to determine the relation to ventricular arrhythmias.

Setting-Investigational laboratory of a tertiary referral centre.

Study design-Patients with left ventricular hypertrophy and normal systolic function $(n=14)$ and patients with chronic heart failure $(n=18)$ were matched with controls $(n=17)$. The QT dispersion was examined in relation to abnormalities in resting mechanical and autonomic function and to the findings of 24 hour Holter monitoring.

Main outcome measures-QT dispersion is the difference between the maximum and the minimum QT values from the 12 lead electrocardiogram. Mean(SD) QT dispersion from the 10 lead electrocardiogram was also examined once the 12 lead minimum and maximum values had been removed. The QT distribution is the curve describing the distance from the mean for all QT intervals (ms).

Results-All measures of QT dispersion were increased significantly in left ventricular hypertrophy and tended to increase in those with heart failure. The QT distribution was abnormal in both heart failure and left ventricular hypertrophy. There was no relation between the degree of change in QT dispersion and the incidence of ventricular arrhythmia on 24 hour Holter monitoring. Also there was no relation between QT dispersion and autonomic or mechanical abnormalities. The QT dispersion was related to QRS duration.

Conclusion-Though QT dispersion and distribution are abnormal in left ventricular hypertrophy these findings do not support the hypothesis that QT dispersion reflects arrhythmic risk in either hypertrophy or heart failure.
\end{abstract}

(Br Heart f 1994;71:268-273)

Ventricular arrhythmias are common in both chronic heart failure, ${ }^{1}$ where they are a leading cause of death, and in left ventricular hypertrophy. ${ }^{23}$ The aetiology of these arrhythmias is likely to be multifactorial ${ }^{4}$ and may include ventricular re-entry. Ventricular re-entry circuits may form on the basis of structural abnormalities that result in a heterogeneous slowing of ventricular depolarisation. After myocardial infarction, and possibly in dilated cardiomyopathy, ${ }^{5}$ these structural abnormalities may be reflected as late potentials on the high gain signal averaged electrocardiogram. Heterogeneous repolarisation may also cause ventricular re-entry. The surface electrocardiogram QT interval reflects duration of myocardial action potential, and it has been proposed that differences in the QT interval between leads, termed QT dispersion, may reflect regional variations in duration of ventricular action potential, and as such may be indicators of arrhythmogenicity. $^{6}$ We therefore hypothesised that in chronic heart failure and in left ventricular hypertrophy-conditions associated with an increased incidence of ventricular arrhythmias-there might be an increase in the surface lead QT dispersion, and that this might reflect the arrhythmic risk.

\section{Patients and methods}

Patients with stable heart failure $(n=18)$, defined as left ventricular dysfunction requiring diuretics for control of symptoms, patients with left ventricular hypertrophy and normal systolic function $(n=14)$ some of whom were on diuretics but only for control of blood pressure, and control patients ( $n=$ 17) who had normal left ventricular dimensions and wall thicknesses were recruited. All patients had QRS complexes of normal duration. Serum electrolytes were all within clinically acceptable limits. Cross sectional and $M$ mode echocardiograms were performed and measurements taken for wall thickness, internal ventricular dimensions at both end diastole (LVIDd) and at end systole (LVIDs), and the index of fractional shortening was derived as (LVIDs/LVIDd). Fractional shortening was used as a measure of the severity of mechanical impairment. Groups were matched for age, weight, and cardiac surgical intervention, except that the hypertrophy group had had less coronary surgery (table 1).

Twelve of the 18 patients with heart failure and 11 of the 17 controls had ischaemic heart disease. Those with heart failure had all had anterior myocardial infarctions. The controls who had ischaemic heart disease as detected by ultrasound had normal left ventricular systolic function and no regional wall motion abnormalities. The hypertrophy group ( $n=$ 14) was either hypertensive $(n=6)$ or had had valve replacement $(n=5)$ and the rest had idiopathic hypertrophy $(n=3)$, although John Radcliffe Hospital, Oxford OX3 9DU.

Accepted for publication 6 September 1993 
Table 1 Demographic details

\begin{tabular}{|c|c|c|c|}
\hline & $\begin{array}{l}\text { Control } \\
(n=18)\end{array}$ & $\begin{array}{l}\text { Hypertrophy } \\
(n=14)\end{array}$ & $\begin{array}{l}\text { Chronic heart failure } \\
(n=17)\end{array}$ \\
\hline $\begin{array}{l}\text { Age }(\mathrm{yr}) \\
\text { Coronary surgery } \\
\text { Frusemide dose }(\mathrm{mg}) \\
\text { QRS duration (ms) } \\
\text { LVIDd(cm) } \\
\text { LVISs }(\mathrm{cm}) \\
\text { Posterior wall }(\mathrm{cm}) \\
\text { LVIDs/LVIDd } \\
\text { BRS ms/mm Hg } \\
\text { [Noradrenaline] pmol// } \\
\text { [Adrenaline] pmol// } \\
\text { Extrasystoles/24h } \\
\text { Patients with VT }\end{array}$ & $\begin{array}{l}59(2) \\
9(50 \%) \\
0 \\
99(2) \\
4 \cdot 6(0 \cdot 1) \\
2 \cdot 9(0 \cdot 2) \\
1 \cdot 0(0 \cdot 04) \\
0 \cdot 61(0 \cdot 04) \\
11 \cdot 3(2 \cdot 1) \\
286(33) \\
207(57) \\
120(66) \\
0\end{array}$ & $\begin{array}{l}56(3) \\
0(0 \%)^{\star \star \star} \\
29(11)^{\star \star \star} \\
103(4) \\
5 \cdot 6(0 \cdot 4) \\
3 \cdot 5(0 \cdot 4) \\
1 \cdot 3(0 \cdot 02)^{\star \star \star} \\
0 \cdot 61(0 \cdot 03) \\
9 \cdot 2(2 \cdot 2) \\
231(64) \\
159(43) \\
1069(492)^{\star} \\
3\end{array}$ & $\begin{array}{l}62(2) \\
7(41 \%) \\
7.6(5)^{\star \star \star} \\
103(3) \\
6 \cdot 1(0 \cdot 2)^{\star \star \star} \\
4 \cdot 8(0 \cdot 3)^{\star \star \star} \\
1 \cdot 1(0 \cdot 06) \\
0 \cdot 79(0 \cdot 01)^{\star \star \star} \\
2 \cdot 8(0 \cdot 2)^{\star \star} \\
448(68)^{\star} \\
212(64) \\
1044(273)^{\star \star \star} \\
1\end{array}$ \\
\hline
\end{tabular}

two patients had been treated for ischaemia. All patients with ischaemia had been fully treated either through percutaneous transluminal coronary angioplasty or through coronary artery grafting, and there were no patients in any of the groups who had either clinical or exercise electrocardiographic evidence of remaining ischaemia. Patients were not on any drugs known to affect the QT interval. The study was approved by the local ethics committee. Informed consent was obtained.

An indwelling venous line was inserted. Standard electrocardiographic leads were attached and patients were rested for $30 \mathrm{~min}$ utes on a bed in a quiet room. Venous samples were then taken for later estimation of catecholamines by high performance liquid chromatography. High speed $(50 \mathrm{~mm} / \mathrm{s})$ and high gain $(20 \mathrm{~mm} / \mathrm{mV})$ electrocardiographic recordings were then taken on a Marquette CASE 15 machine and analysed later. Arterial baroreflex sensitivity was measured by venous injection of incremental doses of phenylephrine, with the arterial pressure continuously measured by a finger Finapress (Ohmeda 2300) device. The baroreflex sensitivity was taken as the slope of the increase in $R R$ interval against the increase in arterial pressure. For each patient the baroreflex sensitivity was measured four times, and the average value of these measurements taken.

Electrocardiographic records were digitised with a SAC (Science Accessories Corporation Graf Pen GP7) digitising tablet connected to a Compaq Deskpro 286e PC. The QT interval was taken from the start of the QRS complex to the end of the $T$ wave. The QT interval was measured from each of the 12 leads of the high speed high gain 12 lead electrocardiograph five times and the data transferred to a Macintosh SC30 computer for further analysis with the Microsoft Excel and the Statview and Graphics software package. The QRS duration was automatically measured by the Marquette CASE 15 .

We attempted to minimise any inaccuracy in the measurement of the QT interval by multiple sampling of each QT interval studied. ${ }^{7}$ Thus for each of the electrocardiographic records we took five measurements of the QT interval from each lead. The arith- metic mean of the QT interval was obtained for each of the 12 leads by averaging the five values. This value was used in all later calculations. The minimum and maximum QT intervals were obtained from the averaged data. The QT dispersion can be defined in a number of different ways. We obtained several different measures. QT 12 lead range was defined as the difference between the mimimum and the maximum QT intervals from the 12 lead data. In those patients with damaged left ventricles low voltage $T$ wave amplitudes are common and may result in poor determination of the end of the $T$ wave. This may result in an important systematic error in the determination of QT dispersion if either the minimum or maximum were affected. Thus we removed the extreme maximum and minimum points to obtain a 10 lead set of data and from this determined QT 10 lead range- that is, the difference between the minimum and the maximum values of QT interval from the 10 lead data set. Maximum and minimum values do not include data on the first 10 and 8 points respectively in each set of data, and it may be that early data points are important. We thus included more of these points by taking the SD of the 12 and 10 lead sets of data, the QT 12 lead SD, and QT 10 lead SD. The data were then reanalysed after a rate correction had been applied to the QT interval by the use of the formula $Q T c=Q T / \sqrt{ } R R$ interval, thus obtaining QTc 12 lead range etc. Finally to correct for intrinsic differences in the QT interval between controls and the other groups the QT 12 lead range was divided into the QT obtained from lead II.

We further examined the possible bias from end point measurement by determining the interval between the maximum and next maximum point and the interval between the minimum and the next point. If there was no significant difference in the different groups between the maximum or minimum point and the next maximum or minimum point it would be unlikely that within group systematic error had occurred at the extremes of QT measurement. This was indeed the case, with the mean distance between the minimum and next point being 14(2) $\mathrm{ms}$ and between the maximum and next point being $22(5)$, with no significant between group difference.

We also considered the distribution of QT intervals around the mean. The time from the 12 lead QTc mean of each lead's QTc interval was obtained, and the modulus derived. The proportion of the set of data contained within $5 \mathrm{~ms}$ batches was then plotted against time from the mean. We also plotted the position of each of the data points from the mean against time from the mean.

Holter monitoring for 24 hours was performed with subsequent analysis for 24 hour frequency of ventricular-extra systoles and episodes of non-sustained ventricular tachycardia.

STATISTICAL ANALYSIS

For between group comparisons 1 and 2 way 
analysis of variance (ANOVA) was used and for direct between variable comparison simple linear regression tests were used. For direct comparison between two groups unpaired $t$ tests were used. Significance was at the $5 \%$ level.

\section{Results}

Table 1 shows patient details. Patients with heart failure received more diuretics, had larger hearts at both end diastole and at end systole, depressed baroreflex sensitivity, and higher noradrenaline concentrations than controls. Both hypertrophy and heart failure groups had more ventricular extrasystoles than controls.

The different QT dispersion measures were related to one another with the 12 and 10 lead QT data being well related to their respective SDs with an $r 0.97$ and $\mathrm{p}<$ 0.0001: thus analysis of the SD provided no extra information than did the range data. The 12 lead data were less well related to the 10 lead data with $r=0.58$ and $p<0.0001$. The QT lead II range data were effectively the same as the 12 lead data with $r=0.98$ and $p<0.0001$. The different measures of QT dispersion all produced the same qualitative results. The mean distance between the minimum and next point was 14(2) ms and between the maximum and next point was 22(5), with no significant between group difference, thus suggesting that end point bias had not occurred.

All measures of QT dispersion were increased (table 3) in left ventricular hypertrophy compared with controls. There was a tendency. for QT dispersion to be increased in heart failure, although this did not reach significance. Not surprisingly, once Bazett's correction was applied the mean dispersion increased in the heart failure group due to a higher mean heart rate, though the comparison with the control group still did not reach significance. When disease classification and position from the mean QT interval were tested against time from the mean QT interval in a two-factor ANOVA, disease classification significantly affected time from the mean QT interval $(p<0.001)$. When patients with hypertrophy or heart failure were separately tested against the control both hypertrophy $(p<0.0001)$ and heart failure $(p<$ 0.02 ) were still separately significant determi-

Table 2 QT dispersion

\begin{tabular}{llll}
\hline & Control & Hypertrophy & CHF \\
\hline QT 12 lead range(ms) & $71(7)$ & $113(14)^{\star \star}$ & $81(8)$ \\
QT 12 lead (SD, ms) & $20(2)$ & $32(4)^{\star \star \star}$ & $23(2)$ \\
Range/QT lead II & $0 \cdot 19(0 \cdot 02)$ & $0 \cdot 30(0 \cdot 05)^{\star \star}$ & $0 \cdot 21(0.02)$ \\
QT 10 lead range(ms) & $43(3)$ & $67(7)^{\star \star \star}$ & $46(4)$ \\
QT 10 lead (SD, ms) & $14(1)$ & $21(2)^{\star \star \star}$ & $15(1)$ \\
$\Delta$ to minimum(ms) & $12(4)$ & $13(3)$ & $15(3)$ \\
$\Delta$ to maximum(ms) & $16(3)$ & $33(13)$ & $20(7)$ \\
QTc 12 lead range $\left(\mathrm{ms}^{-1 / 2}\right)$ & $78(8)$ & $114(14)^{\star}$ & $93(10)$ \\
QTc 12 lead(SD, $\left.\mathrm{ms}^{-1 / 2}\right)$ & $22(2)$ & $33(3)^{\star \star}$ & $26(2)$ \\
QTc 10 lead range $\left(\mathrm{ms}^{-1 / 2}\right)$ & $47(4)$ & $67(6)^{\star \star \star}$ & $52(5)$ \\
QTc 10 lead(SD, $\left.\mathrm{ms}^{-1 / 2}\right)$ & $15(1)$ & $21(2)^{\star}$ & $17(1)$ \\
\hline
\end{tabular}

${ }^{\star} \mathrm{p}<0.05 v$ control; ${ }^{\star \star} \mathrm{p}<0.01 v$ control; ${ }^{\star \star \star} \mathrm{p}<0.005 v$ control. $\Delta$ to minimum, difference between shortest and the next shortest QT interval from 12 lead data; $\Delta$ to maximum, difference between longest and next longest $Q T$ interval from 12 lead data.

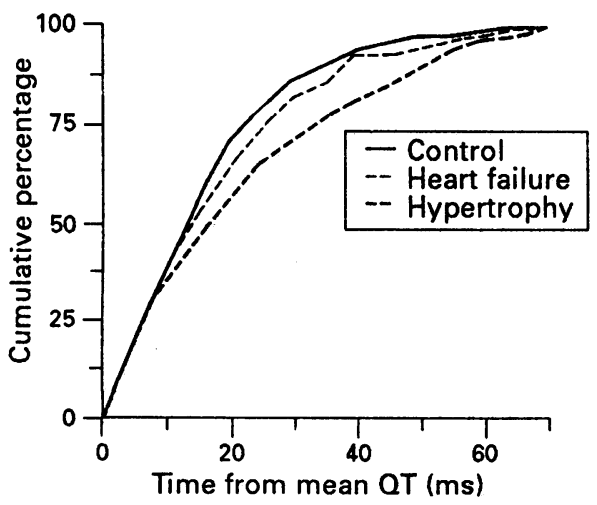

Figure 1 QT distribution around the mean expressed as a cumulative percentage.

nants of time from the mean QT interval. Thus QT intervals around the mean was influenced by left ventricular disease (fig 1). Presence of ischaemic heart disease non-significantly increased measures of QT distribution in both controls and patients with heart failure.

Figure 1 shows the cumulative plot of the difference between QT duration and its mean: though this suggests that the increase in QT dispersion in patients with hypertrophy occurs throughout the range, further analysis showed that this was not the case. The time from the mean that contained $50 \%$ of the data points was not significantly different between the groups $(12.9(1 \cdot 2) \mathrm{ms}$ in controls, $16 \cdot 6(2 \cdot 1) \mathrm{ms}$ in hypertrophy, and $14 \cdot 4(1 \cdot 4)$ $\mathrm{ms}$ in heart failure). Thus the first six QT intervals from each patient's electrocardiogram show an identical pattern spread around the mean QT interval. The separation between the hypertrophy group and the others only reaches significance on the eighth point, and remains significant through to the 12th point (fig 2).

The autonomic and mechanical factors associated with QT dispersion were then examined. Fractional shortening, an index of resting cardiac mechanical function, was not related by linear regression analysis to any of the indices of QT dispersion, although left ventricular end diastolic dimension was related to the 10 lead SD with $r=0.38$ and $\mathrm{p}$ $<0.05$. There was no relation between end

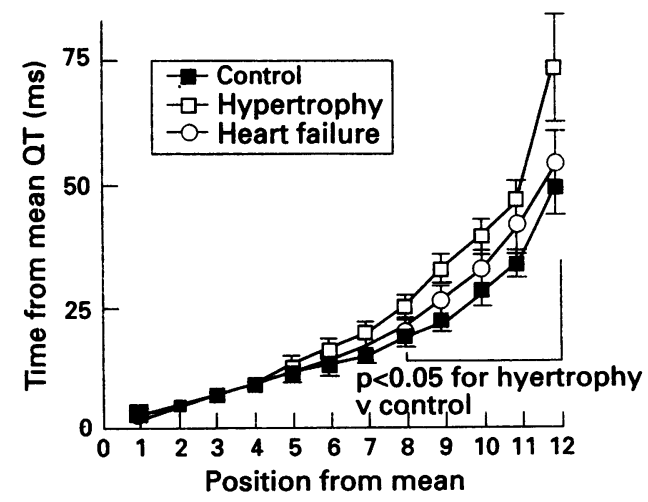

Figure $2, Q T$ distribution around the mean expressed as absolute time. 


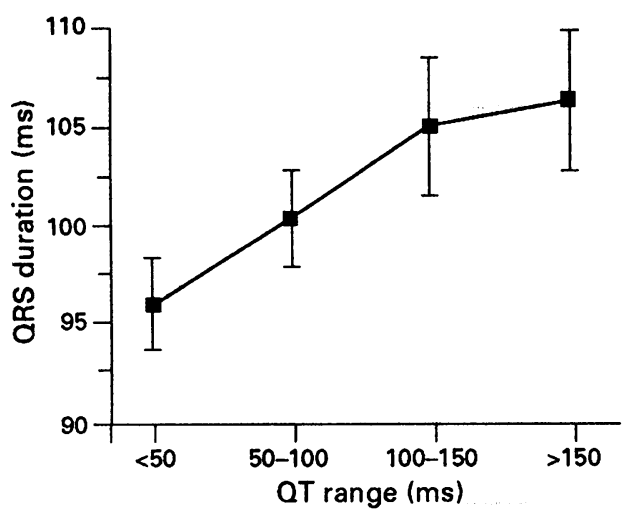

Figure 3 The relation between $Q R S$ duration and $Q T$ (12 lead range).

systolic dimension and any measure of QT dispersion, with the lowest $p$ value $0 \cdot 26$. Although baroreflex sensitivity was also not related to QT dispersion, there was a relation between the 10 lead SD and baroreflex sensitivity $(r=0.318, \mathrm{p}=0.09)$ that approached significance. Resting noradrenaline or resting adrenaline concentrations were not related to QT dispersion. Resting noradrenaline was dichotomised at its mean value and tested against QT dispersion. There was no difference in any measure of QT dispersion between the high and the low noradrenaline group. Likewise when resting adrenaline or baroreflex sensitivity were dichotomised at their mean points there was still no relation with any measure of QT dispersion.

To examine further the relation between QT dispersion and electrical, mechanical, and autonomic dysfunction; the population was dichotomised at $93.8 \mathrm{~ms}$ (the mean point) for the QTc 12 lead range, and was tested against the mechanical, electrical, and autonomic abnormalities. No significant correlation was found except with QRS duration (QTc 12 lead range <93.8, QRS = 98.6(2) ms: QTc 12 lead range > 93.8, QRS $=106(3) \mathrm{ms}, \mathrm{p}<0.03)$. Overall with simple linear regression analysis, although there was a significant relation between QTc 12 lead range and $Q R S$ duration, it was weak with $r=0.34$, and $\mathrm{p}=0.03$ (fig 3).

The relation of QT dispersion to abnormalities on the Holter monitoring was

Table 3 Relation between extrasystolic frequency, mechanical performance, and QT dispersion

\begin{tabular}{|c|c|c|c|}
\hline & $<120 / 24 h$ & $>120 / 24 h$ & p Value \\
\hline $\begin{array}{l}\text { LVIDd(cm) } \\
\text { LVIDs(cm) } \\
\text { LVIDs/LVIDd } \\
\text { BRS(ms/mm Hg) } \\
\text { Resting noradrenaline pmol// } \\
\text { Resting adrenaline pmol// } \\
\text { QT } 12 \text { lead range(ms) } \\
\text { QT } 12 \text { lead(SD, ms) } \\
\text { QT } 10 \text { lead range(ms) } \\
\text { QT } 10 \text { lead(SD, ms) } \\
\text { QT } 12 \text { lead range QT lead II } \\
\text { QTc } 12 \text { lead range( }\left(\mathrm{ms}^{-1 / 2}\right)^{\circ} \\
\left.\text { QT } 12 \text { lead(SD, } \mathrm{ms}^{-1 / 2}\right) \\
\text { QTc } 10 \text { lead range }\left(\mathrm{ms}^{-1 / 2}\right) \\
\left.\text { QTc } 10 \text { lead(SD, } \mathrm{ms}^{-1 / 2}\right)\end{array}$ & $\begin{array}{l}4 \cdot 7(0 \cdot 2) \\
3 \cdot 1(0 \cdot 2) \\
0 \cdot 64(0 \cdot 03) \\
12 \cdot 2(2) \\
311(45) \\
162(36) \\
77(8) \\
22(2) \\
46(4) \\
15(2) \\
0 \cdot 20(0 \cdot 02) \\
83(8) \\
24(2) \\
50(4) \\
16(1)\end{array}$ & $\begin{array}{l}6 \cdot 0(0 \cdot 3) \\
4 \cdot 2(0 \cdot 3) \\
0 \cdot 69(0 \cdot 12) \\
4 \cdot 4(0 \cdot 5) \\
339(46) \\
192(41) \\
93(8) \\
26(2) \\
54(4) \\
17(1) \\
0 \cdot 25(0 \cdot 03) \\
101(9) \\
29(2) \\
58(4) \\
19(1)\end{array}$ & $\begin{array}{l}0.003 \\
0.02 \\
\text { NS } \\
0.002 \\
\text { NS } \\
\text { NS } \\
\text { NS } \\
\text { NS } \\
\text { NS } \\
\text { NS } \\
\text { NS } \\
\text { NS } \\
\text { NS } \\
\text { NS } \\
\text { NS }\end{array}$ \\
\hline
\end{tabular}

Abbreviations as for table 1 . studied. Although the mean number of extrasystoles did not differ between the hypertrophy and heart failure groups, this was largely caused by two patients with hypertrophy who had 5929 and 4054 extrasystoles/24 hours. The mode of extrasystole frequency in the control group was $<120 / 24 \mathrm{~h}$ $(83 \%),<480 / 24 h$ for the hypertrophy group $(71 \%)$, and $>480 / 24 \mathrm{~h}$ for the heart failure group (61\%).

The relation between extrasystole frequency and QT dispersion was examined. With simple linear regression analysis there was no relation between any measure of QT dispersion and extrasystole frequency. When extrasystole frequency was dichotomised at 120 extrasystoles $/ 24 \mathrm{~h}$ (the midpoint of the CAST extrasystolic frequency to mortality dose-response curve) the controls separated from the hypertrophy and heart failure groups $(p<0.001)$ and when dichotomised at 480 extrasystoles $/ 24 \mathrm{~h}$ the heart failure group separated from the other two groups ( $<<$ $0.005)$. As the 480 extrasystoles $/ 24 \mathrm{~h}$ data produced qualitatively similar results to the data of 120 extrasystoles $/ 24 \mathrm{~h}$ only the 120 extrasystoles $/ 24 \mathrm{~h}$ data are presented. The relation of the dichotomised extrasystolic frequency to mechanical impairment, autonomic abnormalities, and the different measures of QT dispersion was studied (table 3). The groups successfully dichotomised on the basis of left ventricular dimensions and baroreflex sensitivity. There were, however, no significant differences in any of the measures of QT dispersion at either of the two levels. There was a trend at the 120 extrasystoles/24 h level for those with the higher extrasystolic frequency to have greater QT dispersion, although this was not significant.

The relation between the presence of nonsustained ventricular tachycardia $(n=4)$ and our measures of QT dispersion was studied. Patients with ventricular tachycardia had significantly larger end diastolic volumes, but did not differ significantly in any measure of QT dispersion.

\section{Discussion}

These data show that QT dispersion is increased in patients with left ventricular hypertrophy, and tends to increase in patients with chronic heart failure. The overall distribution of QT intervals around the mean is altered significantly in patients with left ventricular hypertrophy or heart failure. The QT dispersion has been reported to be increased after myocardial infarction ${ }^{8}$ and unchanged in heart failure without sustained tachycardias. ${ }^{9}$ Different measures of QT dispersion do not seem to correlate well with incidence of arrhythmias on 24 hour Holter monitoring in chronic heart failure, or after myocardial infarction, ${ }^{8}$ or with the frequency of extrasystoles in left ventricular hypertrophy. Others have found that QT dispersion in hypertrophic cardiomyopathy does not relate to the presence of ventricular tachycardias. ${ }^{10}$ Despite the lack of relation with these surrogate 
clinical end points, it is not known whether the increase in QT dispersion may be of pathophysiological or prognostic significance.

The QT interval reflects the duration of the action potential of the ventricular myocytes $^{11}$ and interventions-such as, amiodarone-that alter durations of cellular action potentials also similarly alter the QT interval. Whether QT dispersion accurately reflects regional abnormalities is not yet established. Studies comparing duration of the epicardial action potential with surface lead QT dispersion support this concept ${ }^{12}$ as does the finding that QT dispersion increases in the hereditary long QT syndrome. ${ }^{61213}$ Our finding of increased QT dispersion in patients with left ventricular hypertrophy may reflect the increased range of duration of ventricular action potentials ${ }^{14}$ and activation times. ${ }^{15}$

A relation between $Q T$ dispersion and arrhythmias is not supported by our data. We found no relation between QT dispersion and frequency of extrasystoles, ventricular tachycardia, or the degree of mechanical impairment (an arrhythmic risk factor), ${ }^{16}$ and at best we found a weak relation between QT dispersion and baroreflex sensitivity (an indicator for future arrhythmic events). ${ }^{17}$ Furthermore our finding of a greater QT dispersion in left ventricular hypertrophy than in heart failure, given that the incidence of arrhythmic deaths is higher in chronic heart failure ${ }^{1819}$ than in left ventricular hypertrophy,,$^{20}$ is another argument against duration of dispersion of the action potential being important ${ }^{21}$ in arrhythmogenesis in chronic heart failure.

The absence of a relation between QT dispersion and $24 \mathrm{~h}$ arrhythmias found on Holter monitoring does not necessarily preclude a relation between QT dispersion and subsequent lethal arrhythmias given the relatively low predictive accuracy of 24 hour Holter monitoring. ${ }^{22}{ }^{23}$ Repolarisation changes including increased QT dispersion ${ }^{24}$ during acute myocardial infarction have been related to ventricular fibrillation in the acute phase of myocardial infarction and sustained or inducible monomorphic ventricular tachycardia in the chronic phase. ${ }^{9}$ In hypertrophic cardiomyopathy with inducible polymorphic ventricular tachycardia and subsequent ventricular fibrillation there were differences between the refractory periods of the right ventricular apex and outflow tract ${ }^{26}$ that were thought to be important pathophysiologically. Additional experimental evidence suggests a role for QT dispersion in ventricular arrhythmias complicating intracoronary contrast injections. ${ }^{26}$ In a different experiment a critical dispersion threshold of duration of the action potential of around $100 \mathrm{~ms}^{27}$ was necessary before ventricular arrhythmias could be induced. This may suggest that QT dispersion is important pathophysiologically in the generation of ventricular arrhythmias. If this is the case our finding of an increased QT dispersion in left ventricular hypertrophy might explain the poor prognosis of left ventricular hypertrophy found in community based studies.
The aetiology of the increase in QT dispersion is not clear. The finding of increased QRS duration in those with greater QT dispersion suggests that clinically minor degrees of conducting tissue disease, as is commonly found either in hypertrophy (delayed intrinsicoid deflection) or ischaemic heart disease, might have important consequences for the uniformity of repolarisation. The small increase in the QRS duration $(7 \mathrm{~ms}$ ) would by itself only produce a small increase in QT dispersion: it is therefore likely that the increase in QRS duration is an indicator of the underlying disease process rather than the cause of the increase in QT dispersion. The greater increase in QT dispersion in hypertrophy than in heart failure may be a reflection of changes in basic cellular electrophysiology, some of which (in guinea pig models) ${ }^{28} 29$ are greater in hypertrophy than in heart failure.

It is possible that the increase in QT dispersion is an artefact caused by the difficulty of reading electrocardiograms from diseased hearts. In part we have minimised this by multiple readings of each QT interval. About 3000 digitised QT values were obtained. This technique minimises random but not systematic reading errors: these may occur in heart failure due to flat $T$ waves (underestimate) or in left ventricular hypertrophy due to prominent $U$ waves (overestimate). These reading difficulties are unlikely to affect more than a few leads: thus it was reassuring to see that the 10 lead data produced results that were empirically identical to the 12 lead data, suggesting that this finding was not an artefact. The QT distribution around the mean plot, with deviations consistently greater than the control early on, reaching significance by the eighth data point, also strongly suggest that these findings are not due to spurious end point bias, but represent a more generalised abnormality in QT distribution.

In summary, these data suggest that there is a significant increase in the QT dispersion between leads in left ventricular hypertrophy and a non-significant tendency to increase in chronic heart failure. The QT dispersion did not correlate with indices of mechanical impairment or autonomic function. Also, there seemed to be no connection between increases in QT dispersion and arrhythmia as measured on 24 hour Holter monitoring.

1 Rehnqvist N. Arrhythmias and their treatment in patients with heart failure. Am $\mathcal{f}$ Cardiol 1989;64:61 J-64J.

2 McLenachan JM, Henderson E, Morris KI, Dargie HJ Ventricular arrhythmias in patients with hypertensive left ventricular hypertrophy. $N$ Engl $\Im$ Med 1987;317: 787-92.

3 Ghali JK, Kadakia S, Cooper RS, Liao Y. Impact of left ventricular hypertrophy on ventricular arrhythmias in
the absence of coronary artery disease. $₹ \mathrm{Am}$ Coll Cardiol 1991;17:1277-82.

4 January CT, Riddle JM. Early after depolarisations: mechanisms of induction and block. Circ Res 1989;64: 977-90.

5 Gonska B-D, Bethge K-P, Figulla H-R, Kreutzer $H$. Occurrence and clinical significance of endocardial late potentials and fractionations in idiopathic dilated cardiomyopathy. Br Heart f 1988;59:39-46.

6 Day CP, McComb JM, Campbell RWF. QT dispersion: an indication of arrhythmia risk in patients with long
QT intervals. Br Heart $\mathcal{1 9 9 0 ; 6 3 : 3 4 2 - 4 .}$ 
7 Ahnve S. Errors in the visual determination on corrected QT (QTc) interval during acute myocardial infarction. f Am Coll Cardiol 1985;5:699-702.

8 Dritsas A, Sbarouni E, Oakley CM, Cleland JGF. Is QT interlead variability an arrhythmogenic marker? Br Heart f 1992;68:116.

9 Pye M, Farrell TG, Cobbe SM. QT interval dispersion in patients with sustained ventricular tachycardia or fibrillation. Eur Heart f 1992;13(abstract suppl): 221

10 Linker NJ, Ward I, Griffith MJ, et al. Repolarisation abnormalities in patients with hypertrophic cardiomyopathy and ventricular arrhythmias: are they important? Br Heart ₹ 1992;68:113-4.

11 Olsson SB, Edvardson N, Hirsch I, Blomstrom P Methodological aspects of invasive evaluation of myocardial repolarisation. In: Burrous GS, Schwartz PJ, eds. Clinical aspects of ventricular repolarisation. London: Farrand Press, 1989;67-79.

12 Higham PD, Hilton CJ, Griffiths CJ, Furniss SS, Campbell RWF. Relation between QT intervals and epicardial monophasic action potential. $\mathrm{Br}$ Heart $\mathcal{f}$ 1992;68:123.

13 Linker LJ, Colonna P, Kekwick CA, Till J, Camm J, Ward DE. Assessment of QT dispersion in symptomatic patients with congenital long QT syndrome. $A m f$ patients with congenit

14 Franz MR, Bargheer K, Lichtlen PR, Stinson EB, Miller DC. Myocardial repolarisation in normal and hypertrophied left ventricles. In: Burrous GS, Schwartz PJ, eds. Clinical aspects of ventricular repolarisation London: Farrand Press. 1989;22:219-26.

15 Vassallo JA, Cassidy DM, Kindwall E, Marchlinski FE, Josephson ME Nonuniform recovery of excitability in the left ventricle. Circulation 1988;78:1365-72.

16 Ahnve S, Gilpin E, Henning H, Curtis G, Collins D, Ross J. Limitations and advantages of the ejection fraction for defining high risk after myocardial infarction. $A m \neq 7$ Cardiol 1986;58:872-8.

17 Schwartz PJ, Priori SG. Sympathetic nervous system and cardiac arrhythmias. In: Zipes DP, Jalife J, eds. Cardiac electrophysiology: from cell to bedside. Philadelphia: WB Saunders. 1990;330-43.
18 Cleland JGF, Dargie HJ, Ford I. Mortality in heart failure: clinical variables of prognostic value. $B$ Heart $f$ 1987;58:572-82.

19 Likiff MJ, Chandler SL, Kay HR. Clinical determinants of mortality in chronic heart failure secondary to ideopathic dilated cardiomyopathy or to ischaemic cardiomyopathy. Am $\mathcal{f}$ Cardiol 1987;59:634-8.

20 Cupples LA, Gagnon DR, Kannel WB. Long- and shortterm risk of sudden coronary death. Circulation 1992;85 (suppl I):I-11-8.

21 Janse MJ. The premature beat. Cardiovasc Res 1992;26: 89-100.

22 Packer $M$. Lack of relation between ventricular arrhythmias and sudden death in patients with chronic heart failure. Circulation 1992;85(suppl I):I-50-6.

23 Multicentre Postinfarction Research Group. Risk stratification and survival after acute myocardial infarction. $N$ Engl f Med 1983;309:331-6.

24 Mirvis DM. Spatial variation of QT intervals in norma persons and patients with acute myocardial infarction f Am Coll Cardiol 1985;5:625-31.

25 Higham PD, Campbell RWF. QT Dispersion in ischaemia and infarction. Eur Heart f 1992;14(abstract suppl):448.

26 Watson RM, Schwartz IL, Maron BJ, Tucker E, Rosing DR, Josephson ME. Inducible polymorphic ventricular tachycardia and ventricular fibrillation in a subgroup of tachycardia and ventricular fibrillation in a subgroup of patients with hypertrophic cardiomyopathy at high risk
for sudden death. $₹ \mathrm{Am}$ Coll Cardiol 1987;10:761-74.

for sudden death. F Am Coll Cardiol 1987;10:761-74.
27 Kuo C-S, Munakata K, Reddy P, Surawicz B. Characteristics and possible mechanisms of ventricular arrhythmia dependence on the dispersion of action potential durations. Circulation 1983;67:1356-67.

28 Ryder K, Bryant S, Hart G. Calcium and calcium activated currents in hypertrophied left ventricular myocytes isolated from guinea-pig. 7 Physiol 1990;430. $71 \mathrm{P}-710$.

29 Ryder K, Bryant S, Winterton SJ, Turner MA, Sheridan DJ, Hart G. Electrical and mechanical characteristics of isolated ventricular myocytes from guinea-pigs with left ventricular hypertrophy and congestive heart failure. f Physiol 1991;UC Meeting. 